\title{
On Solving Weakly Singular Volterra Equations of the First Kind with Galerkin Approximations
}

\author{
By John M. Bownds
}

\begin{abstract}
The basic linear, Volterra integral equation of the first kind with a weakly singular kernel is solved via a Galerkin approximation. It is shown that the approximate solution is a sum with the first term being the solution of Abel's equation and the remaining terms computable as components of the solution of an initialvalue problem. The method represents a significant decrease in the normal number of computations required to solve the integral equation.
\end{abstract}

The principal goal here is to show that under typical assumptions on $k$ and $f$ the first kind integral equation

$$
f(x)=\int_{0}^{x} k(x, t)(x-t)^{-\alpha} u(t) d t, \quad 0 \leqslant x \leqslant 1,0<\alpha<1,
$$

can be efficiently solved numerically by using a Galerkin approximation which, essentially, converts the equation into an initial-value problem and approximates the solution as a perturbation of the solution of the classical Abel equation $(k \equiv 1)$. This method, because of the associated significant decrease in computations, relative to other numerical methods, can have the advantage of greater computational speed but the disadvantage of possible increased error. The references supporting these initial comments are found in specific context below.

We assume the usual conditions which imply that (I) has a unique, continuous solution on $[0,1]$, namely that $k(x, t), \partial k(x, t) / \partial x$, and $f^{\prime}(x)$ are continuous on their respective domains, and $k(x, x) \neq 0$ for any $x \in[0,1]$; see [1] , [2]. Without loss, we take $k(x, t)=0$ if $x<t$. This existence theory essentially uses the fact that under these assumptions, (I) is convertible to an equation of the second kind. If $k(x, x)$ $=0$ for some $x$ but $\partial^{p} k(x, x) / \partial x^{p}$ never vanishes for some $p>0$, then certain obvious modifications produce the same existence and uniqueness result, the point being that (I) is still converted to a second kind equation to which the usual fixed point methods can be applied. We assume that $f(0)=0$ and note here that, unfortunately, the smoothness assumption on $f$ is troublesome for some applications involving discrete data; however, this is the same objection which arises with the original Abel inversion of (I) when $k \equiv 1$. See [3] for a modification of the usual inversion which does not explicitly involve $f^{\prime}$. The equivalent second kind equation is as follows.

LEMmA. Under the above assumptions on $k$ and $f$, Eq. (I) is equivalent to the second kind equation

Received September 2, 1975 ; revised October 16, 1975.

AMS (MOS) subject classifications (1970). Primary 45L05; Secondary 45L10, 45H05, 45B05, $45 \mathrm{DO5}$. 


$$
u(x)=\frac{\sin \pi \alpha}{\pi k(x, x)}\left[\frac{d}{d x} \int_{0}^{x}(x-t)^{\alpha-1} f(t) d t+\int_{0}^{x} K(x, t) u(t) d t\right]
$$

where

$$
K(x, t)=-\int_{0}^{1}(1-w)^{\alpha-1} w^{1-\alpha} k_{1}(t+[x-t] w, t) d w
$$

here $k_{1}$ denotes differentiation of $k$ with respect to its first argument. Equivalence, of course, means the two equations have the same solutions on $[0,1]$. This conversion is fairly routine and the actual details are not repeated here.

The approach here is to look for a solution of (I) in the form of a certain sum, the first term of which is the solution of Abel's equation $(k \equiv 1)$ given by the usual inversion. The remaining portion of the proposed solution is formed by solving an initial-value problem; this can represent a major economy due to the excessive number of kernel evaluations $\left(O\left(N^{2}\right)\right)$, where $N$ is the number of tabulations of the solutions encountered in the methods of [1], [4], [5], [14]. This approach has been taken previously in [6], [7] where the main results center on a study of the trade-off between kernel approximation methods, accuracy, and number of computations required to solve second kind Volterra equations with continuous kernels. A generalization of the basic conversion to initial-value problems in [6] - [8] to include Fredholm equations of the second kind is accomplished in [9], again for continuous kernels.

Let $\left\{\phi_{j}\right\}_{j=1}^{\infty}$ be a complete, continuous, orthonormal system in $L^{2}(0,1)$. We seek an approximate solution of (I) of the form

$$
u_{n}(x)=\frac{\sin \pi \alpha}{\pi k(x, x)} \frac{d}{d x} \int_{0}^{x}(x-t)^{\alpha-1} f(t) d t+\sum_{j=1}^{n} \phi_{j}(x) y_{j}(x),
$$

where the functions $y_{j}$ are to be selected in such a way that the error given by

$$
\delta_{n}(x)=u_{n}(x)-\frac{\sin \pi \alpha}{\pi k(x, x)}\left[\frac{d}{d x} \int_{0}^{x}(x-t)^{\alpha-1} f(t) d t+\int_{0}^{x} K(x, t) u_{n}(t) d t\right],
$$

with $K$ as in (1), is orthogonal to the first $n$ basis functions.

THEOREM 1. With the present assumptions, if $\operatorname{col}\left(y_{1}, y_{2}, \ldots, y_{n}\right)$ satisfies the initial-value problem,

$$
y_{j}^{\prime}(x)=\psi_{j}(x) u_{n}(x), \quad 0<x \leqslant 1, \quad y_{j}(0)=0
$$

where

(5) $\psi_{j}(x)=-\frac{\sin \pi \alpha}{\pi} \int_{0}^{1} \int_{0}^{1} \phi_{j}(s)(k(s, s))^{-1}(1-w)^{\alpha-1} w^{1-\alpha} k_{1}(x+[s-x] w, x) d w d s$ and $\phi_{j}, u_{n}$ are as above, then $\left\langle\delta_{n}, \phi_{l}\right\rangle=0$ for $1 \leqslant l \leqslant n ; k_{1}$ again denotes differentiation of $k(x, t)$ with respect to $x$.

Thus, $u_{n}$ is a Galerkin approximation to the solution of (1) provided (4) holds. For linear Fredholm equations of the second kind, the $y_{j}$ 's are constants; and the method is then also referred to as the "method of moments" [10]. Also, in analogy with the classical, linear Fredholm theory [10], [11], Eq. (5) gives the $j$ th coefficient in the Fourier approximation 


$$
\frac{K(x, t)}{k(x, x)} \frac{\sin \pi \alpha}{\pi} \approx \sum_{j=1}^{n} \phi_{j}(x) \psi_{j}(t)
$$

where $K$ is given by (1). Since this Fourier approximation to $K$ is not necessarily most efficient for actually computing the solution of (I), we will prove Theorem 1 as a corollary of the following where we are not as specific about the particular form of the approximation. The theoretical expense is that $\left\langle\delta_{n}, \phi_{l}\right\rangle$ is not zero but, rather, a measure of the deviation of the coefficient $\bar{\psi}_{j}(x)$ in (6) below from the above Fourier coefficient.

Let $K(x, t)$ be given by (1) and assume

$$
\frac{K(x, t) \sin \pi \alpha}{\pi k(x, x)}=\sum_{j=1}^{n} \phi_{j}(x) \bar{\psi}_{j}(t)+\epsilon_{n}(x, t), \quad 0 \leqslant t \leqslant x \leqslant 1,
$$

where $\bar{\psi}_{j}, j=1,2, \ldots, n$, are continuous on $[0,1]$. Notice that since $k(x, t)=0$ if $x<t$, (1) implies $K(x, t)$ also vanishes there. We assume that both terms on the righthand side of (6) vanish for $x<t$.

THEOREM 2. Let $u_{n}$ be given by (2) where col $\left(y_{1}, y_{2}, \ldots, y_{n}\right)$ satisfies (4) with $\psi_{j}$ replaced by $\bar{\psi}_{j}, j=1,2, \ldots, n$. Then

$$
\left\langle\delta_{n}, \phi_{l}\right\rangle=\int_{0}^{1} E_{l}(t) u_{n}(t) d t, \quad 1 \leqslant l \leqslant n,
$$

where

$$
E_{l}(t)=\bar{\psi}_{l}(t)-\frac{\sin \pi \alpha}{\pi} \int_{0}^{1} \frac{\phi_{l}(x) K(x, t)}{k(x, x)} d x, \quad 0 \leqslant t \leqslant 1
$$

Evidently, one might reasonably expect that the approximation used in (6) may produce a better approximate solution to (1) if the magnitude of the right-hand side of (7) is as small as possible. Of course, computationally this may not be particularly easy to verify but it does pose an interesting variational problem. In any case, as we will now see, if $\epsilon_{n}$ in (6) is uniformly small, then $u_{n}$ is near the actual solution; and so, $u_{n}$ is bounded. Also, we record here that $\delta_{n}$ is equivalent to the absolute error $\left(\left|u-u_{n}\right|\right)$ induced by an approximation. We remark here that if $\epsilon_{n} \equiv 0$ (exact decomposition), then it is easy to see that $u_{n} \equiv u$, this being true without orthonormality requirements for $\phi_{1}, \phi_{2}, \ldots, \phi_{n}$.

THEOREM 3. Let $u_{n}, \delta_{n}$, and $\epsilon_{n}$ be as in (2), (3), and (6) respectively; let $\|\cdot\|$ denote the usual sup norm on $[0,1]$. Then

(i) $\left\|u-u_{n}\right\| \rightarrow 0 \Leftrightarrow\left\|\delta_{n}\right\| \rightarrow 0$ as $n \rightarrow \infty$, and

(ii) $\left\|\epsilon_{n}\right\| \longrightarrow 0 \Rightarrow\left\|u-u_{n}\right\| \rightarrow 0$ as $n \rightarrow \infty$; here $\left\|\epsilon_{n}\right\|=\sup \left\{\left|\epsilon_{n}(x, t)\right| ; 0 \leqslant t \leqslant x \leqslant 1\right\}$.

Regarding an estimate on how large the combined truncation and approximation error might be, at worst, since (I) is equivalent to a second kind integral equation, rough a priori error estimates are fairly easy to obtain. Also, however, since the equation is linear, the error due to the approximation (6) can be found with increasing precision by iteration. These two statements form the content of Theorems 4 and 5 .

THEOREM 4. Let $K$ be given by (1) and approximated as in (6). Assume (I) is 
to be solved at the points $x_{k}=k h, k=1, \ldots, N, x_{0}=0, x_{n}=1$, by computing $u_{n}\left(x_{k}\right)=u_{k}$ using (2), where $\operatorname{col}\left(y_{1}, y_{2}, \ldots, y_{n}\right)$ solves (4) with the Fourier coefficient $\psi_{j}$ replaced by $\bar{\psi}_{j}$. If (4) is numerically solved with a method which produces a truncation error not exceeding $\alpha_{k}$ at $x_{k}$, then the combined error due to the approximation in (6) and this truncation error satisfy

$$
e\left(x_{k}\right)=\left|u\left(x_{k}\right)-u_{k}\right|=O\left(\left\|\epsilon_{n}\right\|\right)+\alpha_{k} \text { as }\left\|\epsilon_{n}\right\| \rightarrow 0 .
$$

We note here that this estimate does not include the indicated quadrature in (2). THEOREM 5. Let $v(x)=v_{0}(x)$ denote the solution of the approximating equation

$$
w(x)=\frac{\sin \pi \alpha}{\pi k(x, x)} \frac{d}{d x} \int_{0}^{x}(x-t)^{\alpha-1} f(t) d t+\int_{0}^{x} \sum_{j=1}^{n} \phi_{j}(x) \bar{\psi}_{j}(t) w(t) d t,
$$

$0 \leqslant x \leqslant 1$, and let $u(x)$ denote the solution of (I). Then for $m \geqslant 2$,

$$
u(x)-v(x)=\sum_{j=1}^{m-1} v_{j}(x)+r_{m}(x), \quad 0 \leqslant x \leqslant 1
$$

and

$$
\begin{aligned}
v_{j}(x) & =f_{j}(x)+\sum_{l=1}^{n} \phi_{l}(x) y_{l j}(x) \\
y_{l j}^{\prime}(x) & =\bar{\psi}_{l}(x)\left[f_{j}(x)+\sum_{m=1}^{n} \phi_{m}(x) y_{m j}(x)\right] \\
y_{l j}(0) & =0, \quad l=1,2, \ldots, n ; j=1,2, \ldots, m-1, \\
f_{j}(x) & =\int_{0}^{x} \epsilon_{n}(x, t) v_{j-1}(t) d t, \quad j=1,2, \ldots, m-1,
\end{aligned}
$$

and $\left\|r_{m}\right\|=O\left(C^{m-2} / \sqrt{(m-2) !}\right)$ as $m \rightarrow \infty$; the constant $C$ is the $L^{2}$ norm of

$$
\frac{\sin \pi \alpha}{\pi} \frac{K(x, t)}{k(x, x)} \quad \text { on }[0,1] \times[0,1] \text {. }
$$

We take note of the obvious fact that (10) supplies an infinite series for $u(x)$. The point here is that the above Galerkin approximation is really just the first term in this particular series, and we can refine the approximation by iteration if we desire.

The proofs of Theorems 4 and 5 are supplied in abbreviated form below due to the fact that they have been given elsewhere [6], [7]. Before getting to the details of the first three theorems, we reconsider the approximation (6).

Regarding practical considerations for performing the above calculations, the particular approach taken in a given problem will depend on the properties of the original kernel $k$. It may, for example, be appropriate to use $\psi_{j}$, given by (5), in the kernel approximation (6). In this case, a reasonable outline might be to compute $\psi_{j}(x)$ using Gaussian quadrature as

$$
\psi_{j}(x)=\int_{0}^{1} \sum_{i=1}^{L} H_{i} k_{1}\left(x+[\xi-x] w_{i}, x\right) \phi_{j}(\xi) d \xi
$$


where $w_{i}, H_{i}, i=1,2, \ldots, L$, are zeros and weights associated with the Jacobi polynomials [12]. This takes advantage of the particular form of the right-hand side of (5) in a manner already suggested for Abel's equation in [3]. The remaining integral above could, of course, be found using another appropriate quadrature rule, but this would be appropriate only if the total number of evaluations of the function $k_{1}$ can be kept much smaller than $N^{2}$, where $N$ is the number of solution tabulation points. The advantage seems to be that $\delta_{n}$ is orthogonal to the first $n$ functions of the system $\left\{\phi_{i}\right\}$.

Alternately, it might be advisable to surrender the insistence that (6) be a Fourier approximation and simply approximate the function $k_{1}(x, y)$ with a two-dimensional interpolating polynomial. This approach was used in [6] with reasonable success on smooth equations of the second kind. If this function is approximated by any polynomial as

$$
k_{1}(x, y) \approx \sum_{i+j=0}^{n} \alpha_{i j} x^{i} y^{j}
$$

then, by (1),

$$
K(x, t) \approx-\int_{0}^{1}(1-w)^{\alpha-1} w^{1-\alpha} \sum_{i+j=0}^{n} \alpha_{i j} \sum_{l=0}^{i}\left(\begin{array}{l}
i \\
l
\end{array}\right)(w x)^{l}[t(1-w)]^{i-l} t^{j} d w,
$$

which is just a polynomial with coefficients which are products of beta functions and $\alpha$ 's. The error committed in this interpolation is given in [6] .

Next, we note that if the kernel in the original equation (I) has a derivative $k_{1}$ which decomposes exactly, then the above approximation work is unnecessary. We can, in this case find the solution simply by solving a system like (4) and using (2), which is then an exact equation for the solution. This case will produce a very accurate, fast method for solving (I).

Before showing specific examples, it is noted that all the above development uses $\alpha>0$. Of course, if $\alpha=0$, then the original integral equation has no singularity; and the above assumptions on $k$ easily imply that (I) is equivalent to the equation

$$
u(x)=\frac{f^{\prime}(x)}{k(x, x)}-\int_{0}^{x} \frac{k_{1}(x, t)}{k(x, x)} u(t) d t
$$

and the above results take a much simpler form.

For examples of the above conversion to an initial-value problem, use will be made of examples appearing elsewhere in the literature. The resulting initial-value problems below may, of course, be solved with any number of well-known methods. After some further work on appropriate approximations, the author intends to investigate more complicated and relevant numerical examples in the context of Brownian motion processes as in [5].

Example [14]. The equation

$$
\left(\frac{1}{2}+\frac{4}{5} x^{4}\right) x=\int_{0}^{x}\left(\frac{1}{2}+x^{4}-t^{4}\right) u(t) d t, \quad 0 \leqslant x \leqslant 1,
$$

(exact solution: $u(x) \equiv 1$ ), is of the above form with the simplification that $\alpha=0$. In 
this case the equivalent second kind equation is

$$
u(x)=1+8 x^{4}-8 x^{3} \int_{0}^{x} u(t) d t
$$

and so,

$$
u(x)=1+8 x^{4}-8 x^{3} y(x)
$$

where

$$
y^{\prime}(x)=u(x)=1+8 x^{4}-8 x^{3} y(x), \quad y(0)=0 .
$$

Example [4]. The equation

$$
\left(1+\frac{3}{4} x^{2}\right) x=\int_{0}^{x}(1+x t)(x-t)^{-1 / 2} u(t) d t, \quad 0 \leqslant x \leqslant 1,
$$

(exact solution: $u(x)=2 \sqrt{x} / \pi$ ), is of the required form for the above lemma, and the equivalent second kind equation is

$$
u(x)=\Phi(x)-\frac{1}{\pi\left(1+x^{2}\right)} \int_{0}^{x}\left(\int_{0}^{1}(1-w)^{-1 / 2} w^{1 / 2} t d w\right) u(t) d t
$$

where

$$
\begin{aligned}
\Phi(x) & =\frac{\sqrt{x}}{\pi\left(1+x^{2}\right)}\left(\frac{3}{2} \int_{0}^{1}(1-w) w^{-1 / 2} d w+\frac{21}{8} x^{2} \int_{0}^{1}(1-w)^{3} w^{-1 / 2} d w\right) \\
& =\frac{6 \sqrt{x}}{\pi\left(1+x^{2}\right)}\left(\frac{1}{3}+\frac{2}{5} x^{2}\right)
\end{aligned}
$$

Equation (1) in the above lemma is, in this case, of the form

$$
K(x, t)=-\frac{t}{\pi\left(1+x^{2}\right)} \int_{0}^{1}(1-w)^{-1 / 2} w^{1 / 2} d w=-\frac{B(1 / 2,3 / 2)}{\pi}\left(1+x^{2}\right)^{-1} t,
$$

where $B$ denotes the usual beta function. Hence, since $B(1 / 2,3 / 2)=\pi / 2$, it is true that $K(x, t)=-1 / 2\left(1+x^{2}\right)^{-1} t$, which is exactly decomposable. In accordance with the remark immediately preceding Theorem 3 , we have $u(x)=\Phi(x)-1 / 2\left(1+x^{2}\right) y(x)$, where

$$
y^{\prime}(x)=\left(\Phi(x)-1 / 2\left(1+x^{2}\right)^{-1} y(x)\right) x, \quad y(0)=0 .
$$

Example [4]. The equation

$$
\begin{aligned}
&(2 \pi x)^{-1 / 2} \exp \left(\frac{(1+x)^{2}}{-2 x}\right) \\
& \quad=\int_{0}^{x}(2 \pi)^{-1 / 2}(x-t)^{-1 / 2} \exp \left(-\frac{1}{2}(x-t)\right) u(t) d t, \quad 0 \leqslant x \leqslant 1,
\end{aligned}
$$

[exact solution:

$$
\left.u(x)=\frac{1}{2}(2 \pi x)^{-1 / 2}\left(\exp \left(-\frac{1}{2 x}(1+x)^{2}\right)\left(\frac{1}{x}-1\right)+\exp \left(-2-\frac{1}{2 x}(1-x)^{2}\right)\left(\frac{1}{x}+1\right)\right)\right],
$$

when converted to a second kind equation becomes 


$$
\begin{aligned}
u(x)= & \frac{1}{\pi} \frac{d}{d x} \int_{0}^{x}(x-t)^{-1 / 2} t^{-1 / 2} \exp \left(\frac{(1+t)^{2}}{-2 t}\right) d t \\
& +\frac{1}{2 \pi} \int_{0}^{x}\left(\int_{0}^{1} \exp \left[-\frac{v}{2}(x-t)\right](1-v)^{-1 / 2} v^{1 / 2} d v\right) u(t) d t
\end{aligned}
$$

In this case, the kernel is

$$
K(x, t)=\int_{0}^{1} e^{-(v / 2)(x-t)}(1-v)^{-1 / 2} v^{1 / 2} d v
$$

which requires some approximation. Notice that this integral can be expressed in terms of a confluent hypergeometric function with argument $-1 / 2(x-t)$; in fact, it is only a short exercise to show that

$$
K(x, t)=\frac{\pi}{2} M\left(\frac{3}{2}, 2,-\frac{1}{2}(x-t)\right),
$$

where $M$ is Kummer's function, given by

$$
M(a, b, z)=\sum_{j=0}^{\infty} \frac{(a)_{j}}{(b)_{j}} \frac{z^{j}}{j !}, \quad \cdot(a)_{j}=a(a+1)(a+2) \ldots(a+j-1), \quad(a)_{0}=1 ;
$$

see $[15$, p. 504$]$. So, the kernel can be written as

$$
K(x, t)=\frac{\pi}{2}\left(\sum_{j=0}^{\infty} \gamma_{j}(x-t)^{j}\right)
$$

where

$$
\gamma_{j}=\frac{(3 / 2)_{j}(-1 / 2)^{j}}{(2)_{j} j !}
$$

and this can be written as

$$
K(x, t)=\frac{\pi}{2} \sum_{l=0}^{n} x^{l}\left(\sum_{j=l}^{n} \gamma_{j}\left(\begin{array}{l}
j \\
l
\end{array}\right)(-t)^{j-l}\right)+\epsilon_{n}(x, t) .
$$

If we now express $x^{l}$ as a linear combination of $l$ orthogonal functions $\bar{\phi}_{1}, \ldots, \bar{\phi}_{l}$ on $[0,1]$ (such as, for example, shifted Legendre polynomials), then we obtain

$$
\begin{aligned}
K(x, t) & =\frac{\pi}{2} \sum_{l=0}^{n} \sum_{k=0}^{l} \alpha_{k} \bar{\phi}_{k}(x) \sum_{j=l}^{n} \gamma_{j}\left(\begin{array}{l}
j \\
l
\end{array}\right)(-t)^{j-l}+\epsilon_{n}(x, t) \\
& =\frac{\pi}{2} \sum_{k=0}^{n} \alpha_{k} \bar{\phi}_{k}(x) \sum_{l=k}^{n} \sum_{j=l}^{n} \gamma_{j}\left(\begin{array}{l}
j \\
l
\end{array}\right)(-t)^{j-l}+\epsilon_{n}(x, t),
\end{aligned}
$$

for which in applying Theorem 2, we may take

$$
\phi_{j}(x)=\frac{\pi}{2} \alpha_{j} \bar{\phi}_{j}(x) \text { and } \bar{\psi}_{j}(t)=\sum_{l=j}^{n} \sum_{m=l}^{n} \gamma_{m}\left(\begin{array}{c}
m \\
l
\end{array}\right)(-t)^{m-l} \text {. }
$$

In this case, the approximate solution in Eq. (2) is therefore given by

$$
u_{n}(x)=\frac{1}{\pi} \frac{d}{d x} \int_{0}^{x}(x-t)^{-1 / 2} t^{-1 / 2} \exp \left(\frac{(1+t)^{2}}{-2 t}\right) d t+\sum_{j=1}^{n} \phi_{j}(x) y_{j}(x)
$$


where

$$
y_{j}^{\prime}(x)=\bar{\psi}_{j}(x) u_{n}(x), \quad y_{j}(0)=0
$$

To compute the indicated differentiated integral in this expression, it is probably best to proceed as in [3] and compute

$$
\begin{aligned}
\frac{d}{d x} \int_{0}^{x}(x-t)^{-1 / 2} t^{-1 / 2} \exp \left(\frac{(1+t)^{2}}{-2 t}\right) d t & \\
\approx & \frac{1}{\pi}\left[x^{-1} \exp \left(\frac{(1+x)^{2}}{-2 x}\right)-\frac{1}{2} \sum_{i=1}^{N} H_{i}\left(\frac{f(x)-f\left(x\left[1-u_{i}\right]\right)}{u_{i}}\right)\right]
\end{aligned}
$$

where $f(x)=x^{-1 / 2} \exp (1+x)^{2} /-2 x$ and where the indicated sum 1 to $N$ represents the Gaussian quadrature of the integral

$$
\int_{0}^{1}[f(x)-f(x[1-u])] u^{-3 / 2} d u
$$

the quantities $u_{i}$ and $H_{i}$ are the respective zeros and weights associated with a special version of the Jacobi polynomials. Reference [3] not only contains the details for the quadrature but also lists values for $u_{i}$ and $H_{i}$ in an appendix.

Proof of Theorem 2. Since by the lemma, (I) is equivalent to (II), we use (II) to calculate in the following way.

$$
\begin{aligned}
\left\langle\delta_{n}, \phi_{l}\right\rangle & =\int_{0}^{1}\left(u_{n}(x)-\frac{\sin \pi \alpha}{\pi k(x, x)} \frac{d}{d x} \int_{0}^{x}(x-t)^{\alpha-1} f(t) d t\right. \\
& \left.-\frac{\sin \pi \alpha}{\pi k(x, x)} \int_{0}^{x} K(x, t) u_{n}(t) d t\right) \phi_{l}(x) d x \\
& =\int_{0}^{1}\left(\sum_{j=1}^{n} \phi_{j}(x) y_{j}(x)-\int_{0}^{x}\left(\sum_{j=1}^{n} \phi_{j}(x) \bar{\psi}_{j}(t)+\epsilon_{n}(x, t)\right) u_{n}(t) d t\right) \phi_{l}(x) d x \\
& =\int_{0}^{1}\left(\sum_{j=1}^{n} \phi_{j}(x)\left(y_{j}(x)-\int_{0}^{x} \bar{\psi}_{j}(t) u_{n}(t) d t\right)-\int_{0}^{x} \epsilon_{n}(x, t) u_{n}(t) d t\right) \phi_{l}(x) d x .
\end{aligned}
$$

But, by (4),

$$
y_{j}(x)=\int_{0}^{x} \bar{\psi}_{j}(t) u_{n}(t) d t, \quad 0 \leqslant x \leqslant 1
$$

and so, using (6), we have

$$
\begin{aligned}
\left\langle\delta_{n}, \phi_{l}\right\rangle & =-\int_{0}^{1} \phi_{l}(x)\left(\int_{0}^{x}\left[\frac{K(x, t) \sin \pi \alpha}{\pi k(x, x)}-\sum_{j=1}^{n} \phi_{j}(x) \bar{\psi}_{j}(t)\right] u_{n}(t) d t\right) d x \\
& =-\int_{0}^{1}\left(\int_{t}^{1} \frac{\phi_{l}(x) K(x, t) \sin \pi \alpha}{\pi k(x, x)} d x-\int_{t}^{1} \phi_{l}(x) \sum_{j=1}^{n} \phi_{j}(x) d x \bar{\psi}_{j}(t)\right) u_{n}(t) d t .
\end{aligned}
$$

Now, because all three terms in (6) are zero when $x<t$, this can be written as

$$
\left\langle\delta_{n}, \phi_{l}\right\rangle=-\int_{0}^{1}\left(\frac{\sin \pi \alpha}{\pi} \int_{0}^{1} \frac{\phi_{l}(x) K(x, t)}{k(x, x)} d x-\bar{\psi}_{l}(t)\right) u_{n}(t) d t,
$$

where we have used the orthonormality of the $\phi$ 's and the assumption that $1 \leqslant l \leqslant n$. This completes the proof; Theorem 1 follows as a corollary in a straightforward fashion recalling (1). 
Proof of Theorem 3. If $f_{i}$ and $K_{i}$ are continuous, and

$$
w_{i}(x)=f_{i}(x)+\int_{0}^{x} K_{i}(x, t) w_{i}(t) d t, \quad i=1,2,
$$

then if $\left|K_{1}(x, t)-K_{2}(x, t)\right| \leqslant \epsilon$ for all $0 \leqslant t \leqslant x \leqslant 1$, it follows that

$$
\left\|w_{1}-w_{2}\right\| \leqslant\left(\left\|f_{1}-f_{2}\right\|+\epsilon\left\|w_{2}\right\|\right) \exp \left(\left\|K_{1}\right\|\right)
$$

where $\left\|K_{1}\right\|$ is the maximum of $K_{1}(x, t)$ on $0 \leqslant t \leqslant x \leqslant 1$. This follows from an elementary application of Gronwall's inequality or any number of treatises on Volterra equations; see [13]. Now, the assumptions on $K$ are sufficient to deduce from (3), that $\left\|\delta_{n}\right\| \rightarrow 0$ if $u_{n} \rightarrow u$ uniformly in view of (II). On the other hand, if we take

$$
f_{1}(x)=\frac{\sin \pi \alpha}{\pi k(x, x)} \frac{d}{d x} \int_{0}^{x}(x-t)^{\alpha-1} f(t) d t, \quad f_{2}(x)=\delta_{n}(x)+f_{1}(x),
$$

and $K_{1} \equiv K_{2} \equiv K$, we see from the above comparison lemma that $\left\|u-u_{n}\right\| \leqslant$ $\left\|\delta_{n}\right\| \exp (\|K\|)$, from which the converse follows, and so (i) is proved.

For (ii), taking $f_{1}$ as above, $f_{2} \equiv f_{1}$,

$$
K_{1}(x, t)=\frac{\sin \pi \alpha}{\pi k(x, x)} K(x, t)
$$

and

$$
K_{2}(x, t)=\sum_{j=1}^{n} \phi_{j}(x) \bar{\psi}_{j}(t), \quad 0 \leqslant t \leqslant x,
$$

the above inequality implies that

$$
\left\|u-u_{n}\right\| \leqslant\left\|\epsilon_{n}\right\| \exp \left(\left\|K_{1}\right\|\right)
$$

from which the result follows immediately. The proofs of Theorems 4 and 5 are given in [6], [7]; however, since these references are not yet in print, they will be at least sketched here.

Proof of Theorem 4. If $v\left(x_{k}\right)$ denotes the exact solution at $x_{k}$ of the approximating equation (9), then $\hat{u}_{k}$ is the numerical approximation of $v\left(x_{k}\right)$. This follows from the representation theorem in [6], [7] which, basically, asserts that if the kernel in a Volterra equation is of the form in (9), then the exact solution is of the form given in (2); no approximation is needed. (We are, as mentioned above, not considering the error involved in the indicated quadrature in (2).) Then, writing

$$
e\left(x_{k}\right)=\left|u\left(x_{k}\right)-\hat{u}_{k}\right| \leqslant\left|u\left(x_{k}\right)-v\left(x_{k}\right)\right|+\left|v\left(x_{k}\right)-\hat{u}_{k}\right|,
$$

the result easily follows using Gronwall's inequality together with other routine estimates.

Proof of Theorem 5. Let $u(x)$ and $v(x)$ solve (II) and therefore (I) and (9), respectively. Then

$$
u(x)-v(x)=\int_{0}^{x} \kappa(x, t)(u(t)-v(t)) d t+\int_{0}^{x} \epsilon_{n}(x, t) v(t) d t
$$

where

$$
\kappa(x, t)=\frac{\sin \pi \alpha K(x, t)}{\pi k(x, x)}
$$


Letting $r_{1}(x)=u(x)-v(x)$, we see that $r_{1}$ satisfies the original Eq. (II) with

$$
\frac{\sin \pi \alpha}{\pi k(x, x)} \frac{d}{d x} \int_{0}^{x}(x-t)^{\alpha-1} f(t) d t
$$

replaced by

$$
f_{1}(x)=\int_{0}^{x} \epsilon_{n}(x, t) v(t) d t
$$

Proceeding in this fashion, defining $r_{m}(x)=r_{m-1}(x)-v_{. m-1}(x)$, and $f_{j}, v_{j}$ as in the theorem, it is reasonably straightforward to establish (10).

To complete the proof, we note that for $m \geqslant 2$,

$$
f_{m}(x)=\int_{0}^{x} \epsilon_{n}(x, t)\left[f_{m-1}(t)+\sum_{l=1}^{n} \phi_{l}(t) y_{k, m-1}(t)\right] d t,
$$

from which it can be shown that

$$
f_{m}(x)=\int_{0}^{x} S(x, t) f_{m-1}(t) d t
$$

for a certain continuous function $S$. But this defines a sequence of Picard iterates for the equation

$$
g(x)=\int_{0}^{x} S(x, t) g(t) d t
$$

which, by the continuity of $S$, has only $g \equiv 0$ as solution. Thus $f_{m} \rightarrow 0$ uniformly. Then, since $r_{m}$ satisfies

$$
r_{m}(x)=f_{m}(x)+\int_{0}^{x} \kappa(x, t) r_{m}(t) d t,
$$

it follows that $r_{m} \rightarrow 0$ uniformly, this again making use of the usual Gronwall or comparison lemma. To see that $r_{m}$ actually satisfies the indicated estimate requires an adaption of an argument found in [11, p. 12]; the main details of this adaption are in [6].

Department of Mathematics

University of Arizona

Tucson, Arizona 85721

1. R. WEISS, "Product integration for the generalized Abel equation," Math. Comp., v. 26, 1972, pp. 177-190. MR $45 \# 8050$.

2. G. KOWALEWSKI, Integralgleichungen, de Gruyter, Berlin, 1930.

3. H. FETTIS, "On the numerical solution of equations of Abel type," Math. Comp., v. 18, 1964, pp. 491-496. MR 30 \#2696.

4. R. WEISS \& R. S. ANDERSSEN, "A product integration method for a class of singular first kind Volterra equations," Numer. Math., v. 18, 1971/72, pp. 442-456. MR 47 \#1314.

5. R. S. ANDERSSEN, F. R. de HOOG \& R. WEISS, "On the numerical solution of Brownian motion processes," J. Appl. Probability, v. 10, 1973, pp. 409-418. MR 50 \#3374.

6. J. BOWNDS \& B. WOOD, "On numerically solving nonlinear Volterra integral equations with fewer computations," SIAM J. Numer. Anal., v. 13, 1976.

7. J. BOWNDS \& B. WOOD, "A faster numerical method for solving Volterra integral equations with convolution kernels." (Submitted.)

8. J. M. BOWNDS \& J. M. CUSHING, "A representation formula for linear Volterra integral equations," Bull. Amer. Math. Soc., v. 79, 1973, pp. 532-536. MR 47 \#2285.

9. M. GOLDBERG, "The conversion of Fredholm integral equations to equivalent Cauchy problems," Appl. Math. Comput. (To appear.) 
10. L. V. KANTOROVIČ \& V. I. KRYLOV, Approximate Methods of Higher Analysis, 3rd ed., GITTL, Moscow, 1950; English transl., C. Benster, Interscience, New York; Noordhoff, Groningen, 1958. MR 13, 77; 21 \#5268.

11. F. G. TRICOMI, Integral Equations, Pure and Appl. Math., vol. 5, Interscience, New York and London, 1957. MR 20 \#1177.

12. G. SZEGÖ, Orthogonal Polynomials, Amer. Math. Soc. Colloq. Publ., vol. 23, Amer. Math. Soc., Providence, R. I., 1939. MR 1, 14.

13. T. SATŌ, "Sur l'équation intégrale nonlinéaire de Volterra," Compositio Math., v. 11, 1953, pp. 271-290. MR 15, 714.

14. F. R. de HOOG \& R. WEISS, "High order methods for Volterra integral equations of the first kind," SIAM J. Numer. Anal., v. 10, 1973, pp. 647-658.

15. M. ABRAMOWITZ \& I. A. STEGUN, Editors, Handbook of Mathematical Functions, With Formulas, Graphs, and Mathematical Tables, Nat. Bur. Standards Appl. Math. Series, vol. 55, Supt. of Documents, U. S. Govt. Printing Office, Washington, D. C., 1964. MR 29 \#4914. 